\title{
A sequence of critical temperatures in YBCO between 20 and $70 \mathrm{~K}$
}

\author{
K N R TAYLOR, G J RUSSELL, S BOSI, S L TOWN, \\ B HUNTER and C N W DARLINGTON* \\ Advanced Electronic Materials, School of Physics, University of New South Wales, P.O. Box \\ 1, Kensington, N.S.W. 2033, Australia. \\ * School of Physics and Space Science, University of Birmingham, Birmingham, B15 2TT, \\ England.
}

\begin{abstract}
Evidence has been found for a number of discrete and sharp transitions in the magnetic susceptibility transition of many single crystals of YBCO. These appear to favour certain temperatures and at least eight transitions are observed between $20 \mathrm{~K}$ and $70 \mathrm{~K}$. One of these transitions dominates the behaviour of one of the individual crystals and X-ray studies indicate that the oxygenation value corresponds to $\mathrm{O}_{6.7}$. We suggest that these transitions are associated with different ordered states in the oxygen deficient lattice.
\end{abstract}

Keywords. Critical temperatures; YBCO; oxygen defect ordering.

\section{Introduction}

The strong dependence of the superconducting behaviour of the $\mathrm{Y}-\mathrm{Ba}-\mathrm{Cu}$ oxide perovskite materials is well known and arises from effects associated with the population of the $\mathrm{O}_{4}$ and $\mathrm{O}_{5}$ oxygen sites in the structure (Jorgensen et al 1987). With complete oxygenation i.e. $\mathrm{YBa}_{2} \mathrm{Cu}_{3} \mathrm{O}_{7}(\delta=0)$, selective occupation of the $\mathrm{O}_{4}$ site occurs and the $\mathrm{O}-\mathrm{Cu}-\mathrm{O}$ chains form. This anisotropic filling leads to a lattice distortion which results in the orthorhombic superconducting phase and also in the optical domain and twin structures which are important characteristics of the (123) materials.

With decreasing oxygen concentration, oxygen atoms are removed selectively from the chain sites and theoretical modelling shows that a wide range of different ordering states become possible (de Fontaine et al 1987) and evidence for such local ordering has been reported by several workers (see for example, van Tendeloo et al 1987). The best known example corresponds to the $\mathrm{O}_{6.5}$ stoichiometry where the oxygen chain structure develops a superlattice phase with alternate chains occupied and intermediate chains empty. This $2 \mathrm{a}, \mathrm{b}$, c structure is well-correlated with the $60 \mathrm{~K}$ superconducting phase first reported by Cava et al (1987). Some evidence for other distinct phases has been reported recently by Schwartz et al (1989) and ourselves (Bosi et al 1989a).

It might be anticipated that a range of stable superconducting phases may exist in the (123) compounds, each one associated with one of the various stable oxygen ordered states which have been reported. It is still unclear, however, whether the more complex ordered states such as the $2 / 3$ (two full chains, one empty) or $1 / 3$ structures, both of which give a superlattice phase $3 a, b, c$, can be stabilized over large enough volumes for the corresponding superconducting phase to appear. Clearly the possibility of observing such new phases depends upon the extent of the ordering as well as upon the detailed mechanisms responsible for the superconduction process in these oxides.

At present, the processing conditions necessary to stabilize the different oxygen phases are not known, and if other superconducting phases are to be found it is likely 
that they will be observed serendipitously during measurements on materials processed using non-normal methods. Our earlier report (Bosi et al 1989a), which was based on observations of large single crystals in which the oxygen distribution was known to be highly inhomogeneous, encouraged us to carry out a systematic search for anomalous transitions in these crystals. The results of this study are given in the following.

\section{Experimental}

All the observations were carried out on single crystals grown from a chloride flux using the method reported by Bosi et al (1989b) recently. The individual crystals were cleaved from polycrystalline boules and examined in their as-grown state. A.c. susceptibility was measured using a conventional balanced inductance bridge, with a drive field of $5 \mathrm{mOe}$ at $1000 \mathrm{~Hz}$. The temperature variation of both the real and complex components was recorded for each crystal over the temperature range $4-100 \mathrm{~K}$ with a temperature resolution of $0.5 \mathrm{~K}$, and where anomalies were observed the appropriate interval was re-examined with a resolution of $0.1 \mathrm{~K}$.

An example of the magnetic transition of a typical crystal is shown in figure 1 in which can be seen a large number of individual step-like changes in $\chi^{\prime}$ as the material

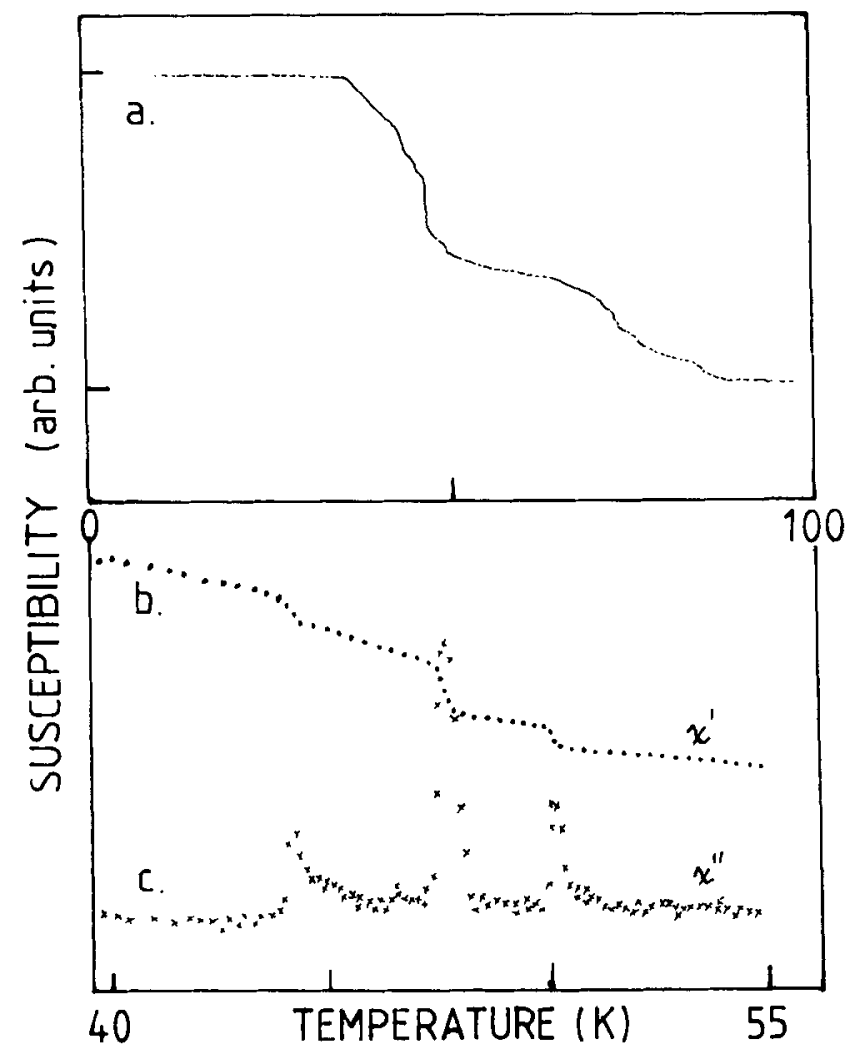

Figure 1. a. Superconducting transition of one of the crystals examined, showing the step-like behaviour. Expanded data, at higher temperature resolution for $\mathbf{b}$. the real part and $\mathbf{c}$. the complex part of the a.c. susceptibility. 
becomes superconducting. The corresponding changes in $\chi^{\prime \prime}$ suggest that there are sharp loss peaks associated with at least some of the $\chi^{\prime}$ steps. Re-examination of the temperature range between 40 and $55 \mathrm{~K}$ at a higher resolution showed that three welldefined transitions could be observed, each one with a corresponding loss peak. As may be seen from figure 1 the width of these peaks is less than $0.5 \mathrm{~K}$ and consequently reflect extremely well-defined physical processes.

The observation of such transitions is relatively common, occurring in approximately half of all crystals studied. Surprisingly, however, the temperatures at which the transitions appear vary considerably from specimen to specimen as also does the amplitude of the individual transitions in both $\chi^{\prime}$ and $\chi^{\prime \prime}$. Examples of intermediate transitions associated with other crystals are shown in figure 2 from which it is clear that good experimental control is necessary to ensure that these anomalous transitions are adequately resolved. Using a temperature resolution of $0.1 \mathrm{~K}$, observations were made on a number of crystals for which the preliminary susceptibility measurements had suggested the presence of intermediate transitions. The various transition temperatures $\left(T^{*}\right)$ associated with these crystals were subsequently plotted in the form of a histogram as shown in figure 3. It is clear from this figure that the individual $T^{*}$ values are not rar.domly spread in temperature, but rather favour a number of discrete values varying from a lower limit of $20 \mathrm{~K}$ to an upper limit of just greater than $70 \mathrm{~K}$. There is also evidence for a spread of discrete critical temperature values close to $90 \mathrm{~K}$ (Bhargava et al 1989) but the nature of these will not be discussed in the present work. The resolution of this histogram corresponds to a bin size of $2.5 \mathrm{~K}$ and it is clear by comparison with figure 2 that this is only barely sufficient to resolve some of the $T^{*}$ transitions lying in the temperature ranges $38-42 \mathrm{~K}$ and $44-50 \mathrm{~K}$. With the limited data

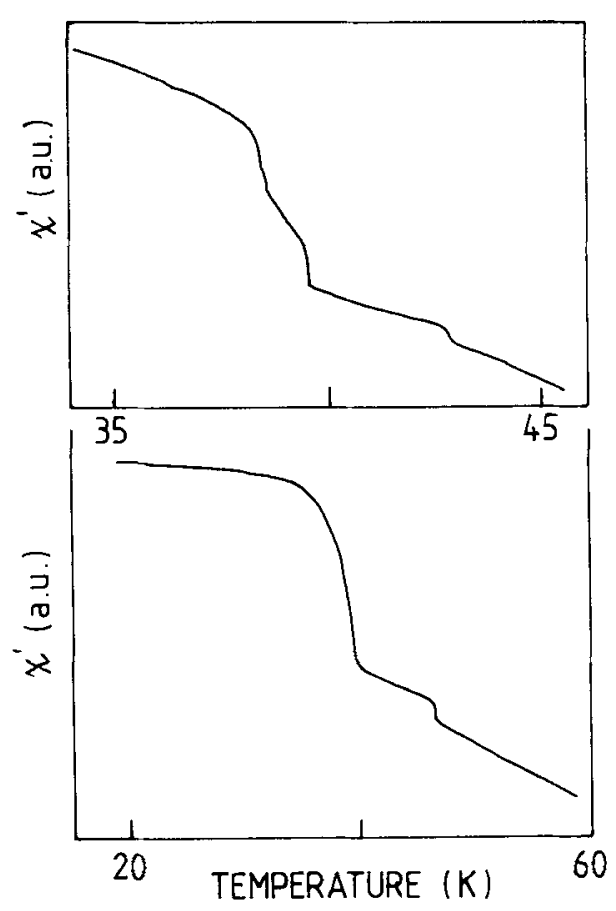

Figure 2. Examples of discrete transitions in other specimens. 


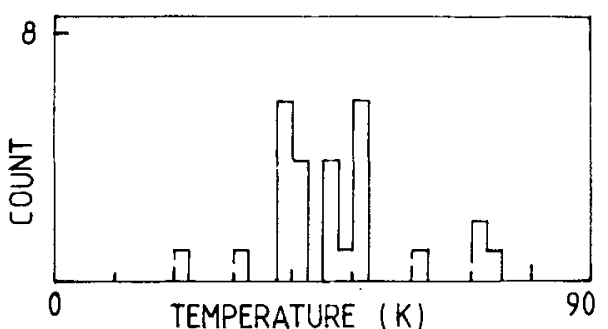

Figure 3. Histogram of the observed $T^{*}$ values.

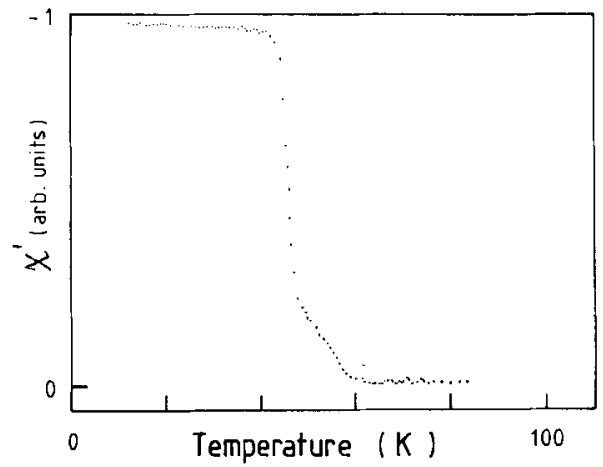

Figure 4. An a.c. susceptibility transition dominated by a $T^{*}$ of approximately $45 \mathrm{~K}$.

available to us at present, however, we would not wish to impose a tighter restriction on figure 3 and have indicated the values of $T^{*}$ which we believe to be well established in these observations.

While majority of the transitions shown in figure 3 are associated with relatively small steps in the observed susceptibility, this is not always the case and figure 4 shows the full $\chi^{\prime}(T)$ transition for one of the smaller crystals used in the study. While the overall transition clearly shows an onset at $60 \mathrm{~K}$ the major part of the transition occurs at $45 \mathrm{~K}$ over a narrow $(<1 \mathrm{~K})$ temperature range. This temperature corresponds to one of the discrete transitions indicated in figure 3 and we believe that in this crystal the processing conditions have been such as to favour the stabilization of this particular phase. Optical polarimetric microscopy of the crystal shows clearly that this specimen is a bicrystal with a small piece of a second crystal attached to one side of the main crystal. This had obviously come away from the boule during cleaving and as yet we have not attempted to remove this second component. It is possible that the two transitions of this specimen are associated with the two components.

$\mathrm{X}$-ray observations of the crystal using the Weissenberg technique show the presence of this second component and also have allowed us to obtain a measure of the orthorhombic distortion. For both components this is close to $(b-a) / a=0.0146$ and may be used to obtain an estimate of the oxygen concentration as $\mathrm{O}_{6 \cdot 7}$.

\section{Discussion}

It is clear from the results described above that there is considerable evidence for the existence of a range of transitions in the temperature dependence of the a.c. susceptibility of single crystals of YBCO. It is also clear that under certain conditions the processes which give rise to these transitions may be stabilized to favour one or other of the observed $T^{*}$ values. Since under the conditions of preparation of these crystals the most likely variable is the oxygen concentration it is tempting to assume that we are seeing various stable superconducting phases associated with different ordered states of the oxygen vacancy system. If this is so then it would suggest that the $45 \mathrm{~K}$ transition of figure 4 may be related to the $2 / 3$ chain phase referred to earlier, since this corresponds to the oxygen concentration of $\mathrm{O}_{6 \cdot 7}$ measured in this specimen. It is well known that the $60 \mathrm{~K}$ phase also appears in this oxygen concentration region and it 
is possible that the processing conditions for this crystal have favoured the stabilization of the $2 / 3$ phase rather than a combination of the 60 and $90 \mathrm{~K}$ phases. If this interpretation is correct it is of considerable importance that the critical temperature of this phase is lower than that of the half-filled chain system $(60 \mathrm{~K})$ since it suggests that the structure of the ordered oxygen system may dominate over the effects of the number of carriers in the solids.

\section{Conclusions}

As a result of a survey of the magnetic transitions in a number of superconducting single crystals of $\mathrm{YBCO}$ evidence has been found for a range of discrete critical temperatures which may be associated with the different ordered states of the oxygen lattice in incompletely oxygenated material. In one crystal a transition close to $45 \mathrm{~K}$ dorninated the behaviour and the $\mathrm{X}$-ray measurement of the oxygen concentration as $\mathrm{O}_{6.7}$ suggests that this may be related to the $2 / 3$ chain structure.

\section{References}

Bosi $S$ et al 1989a Physica $\mathbf{C}$ (to be published)

Bosi S, Puzzer T, Hunter B, Russell G J, Town S L and Taylor K N R 1989b J. Mater. Sci. Letts. 8497 Cava R J, Batlogg B, Chen C H, Rietman E A, Zahurak S M and Werder D 1987 Nature (London) 329423 de Fontaine D, Willie L T and Moss S C 1987 Phys. Rev. B36 5709

Jorgensen J D et al 1987 Phys. Rev. B36 3608

Schwartz M, Rappaport M, Hodes G, Reich S and Cahen D 1989 Mater. Letts. 7411

van Tendeloo G, Zandbergen H W and Amelinckx S 1987 Solid State Commun. 63 298, 606 